\title{
CB Research Square \\ Progress and Challenges of Integrated Drug Efficacy Surveillance for Uncomplicated Malaria in Thailand
}

\author{
Prayuth Sudathip \\ DVBD \\ Aungkana Saejeng \\ DVBD \\ Nardlada Khantikul \\ ODPC 1 \\ Thannikar Thongrad \\ DVBD \\ Suravadee Kitchakarn \\ DVBD \\ Rungniran Sugaram \\ DVBD
}

Cheewanan Lertpiriyasuwat

DVBD

\section{Darin Areechokchai}

DVBD

Deyer Gopinath

WHO: Organisation mondiale de la Sante

David Sintasath

USAID

Pascal Ringwald

WHO: Organisation mondiale de la Sante

Sathapana Naowarat

RTI International

Niparueradee Pinyajeerapat

USAID

Maria Dorina Bustos

WHO: Organisation mondiale de la Sante

Jui A. Shah ( $\nabla$ juishah@rti.org )

RTI International https://orcid.org/0000-0001-5723-4738

\section{Research Article}

Keywords: Malaria elimination, surveillance, drug efficacy, antimalarial, drug resistance.

Posted Date: March 25th, 2021

DOI: https://doi.org/10.21203/rs.3.rs-343048/v1

License: (c) (1) This work is licensed under a Creative Commons Attribution 4.0 International License. Read Full License 


\section{Abstract}

\section{Background}

Integrated drug efficacy surveillance (iDES) was formally introduced nationally across Thailand in fiscal year 2018 (FY2018), building on a history of drug efficacy monitoring and interventions. According to the National Malaria Elimination Strategy for Thailand 2017-2026, diagnosis is microscopically confirmed, treatment is prescribed, and patients are followed up four times to ensure cure.

\section{Methods}

Routine patient data were extracted from the malaria information system for FY2018-FY2020. Treatment failure of first-line therapy was defined as confirmed parasite reappearance within 42 days for Plasmodium falciparum and 28 days for Plasmodium vivax. The primary outcome was the crude drug efficacy rate, estimated using Kaplan-Meier methods, at day 42 for $P$. falciparum treated with dihydroartemisinin-piperaquine plus primaquine, and day 28 for $P$. vivax treated with chloroquine plus primaquine; day 60 and day 90 efficacy were secondary outcomes for $P$. vivax.

\section{Results}

The proportion of patients with outcomes recorded at day 42 for $P$. falciparum malaria and at day 28 for $P$. vivax malaria has been increasing, with FY2020 follow-up rates of $61.5 \%$ and $57.2 \%$, respectively. For $P$. falciparum malaria, day 42 efficacy in FY2018 was 92.4\% ( $n=249)$, in FY2019 93.3\% $(n=379)$, and in FY2020 98.0\% ( $n=167)$. P. falciparum recurrences occurred disproportionally in Sisaket Province, with day 42 efficacy rates of $75.9 \%$ in FY2018 ( $n=59)$ and 49.4\% in FY2019 ( $n=49)$, leading to an update in first-line therapy to pyronaridine-artesunate at the provincial level, rolled out in FY2020. For $P$. vivax malaria, day 28 efficacy was 98.5\% in FY2018 ( $n=2,048), 99.1 \%$ in FY2019 ( $n=2,206)$, and 99.9\% in FY2020 ( $n=2,448)$, and day 90 efficacy was $94.8 \%, 96.3 \%$, and $97.1 \%$, respectively.

\section{Conclusions}

In Thailand, iDES provided operationally relevant data on drug efficacy, enabling the rapid amendment of treatment guidelines to improve patient outcomes and reduce the potential for the spread of drug-resistant parasites. A strong case-based surveillance system, integration with other health system processes, supporting biomarker collection and molecular analyses, and cross-border collaboration may maximize the potential of iDES in countries moving towards elimination.

\section{Background}

The Greater Mekong Subregion (GMS) is the global epicenter of antimalarial drug resistance. High failure rates for artemisininbased combination therapies (ACTs) against Plasmodium falciparum have been reported throughout the region, associated with resistance to both artemisinins and their partner drugs [1]. In 2008, through the Containment Project, the World Health Organization (WHO) and its partners, with extra funding from the Bill \& Melinda Gates Foundation, promoted a policy of containment and eventual elimination of artemisinin-resistant $P$. falciparum in the Thailand - Cambodia border area [2]. This strategy was subsequently expanded to target malaria elimination across the GMS for $P$. falciparum by 2025 and all malaria by 2030 [3].

The National Malaria Elimination Strategy for Thailand 2017-2026 envisions the elimination of malaria by 2024 [4, 5]. Thailand is situated between two discrete malaria transmission regions, with the north-east provinces in a zone comprising Cambodia, southern Vietnam, and southern Laos; and the western provinces in a zone with eastern Myanmar and northern Malaysia. Although $P$. falciparum and $P$. vivax are the dominant parasites, malaria cases caused by $P$. malariae, $P$. ovale, and $P$. knowlesi also occur, as do mixed infections. Malaria transmission in Thailand is concentrated in forested areas along these international borders, with forest workers, displaced people, refugees, migrants, and police and military personnel most exposed to risk [6]. High population mobility in these areas can cause malaria to re-establish in villages where the disease has been eliminated, and civil unrest in the south of the country has hampered malaria control activities [7]. Despite these challenges, 
malaria incidence has declined overall since 2000 , though more rapidly for $P$. falciparum compared with $P$. vivax [8]. $P$. vivax clinical resistance to chloroquine has been reported rarely in Thailand [9]. However, $P$. vivax is refractory to standard malaria control interventions, partly because of the persistence of $P$. vivax hypnozoites, which are undetectable and can remain dormant for weeks or months before reactivation [10].

Targeting malaria elimination has required repositioning of the health system in Thailand. At a national level, the Department of Disease Control is responsible for malaria policy and strategy, whereas the Division of Vector Borne Diseases (DVBD) undertakes capacity building and provides technical support, including managing the Malaria Information System (MIS) - a national database for malaria surveillance and monitoring [11]. The electronic MIS was initially designed to track artemisinin resistance while also modernizing Thailand's existing paper-based reporting system [2]. In addition to malaria diagnosis and treatment, case investigation is conducted to determine the origin of the infection, thereby enabling identification, classification, and elimination of transmission foci [12]. In areas deemed to be at risk, active case detection is employed and vector control measures enhanced, with the aim of interrupting transmission [4]. This case-based malaria surveillance is captured in the MIS, providing near real-time information, which the DVBD uses to stratify malaria risk down to the village level. These detailed and timely data allow local authorities to understand the potential for malaria outbreaks, and to respond quickly should they occur [4].

Therapeutic efficacy studies (TES) have been the primary tool deployed to track antimalarial efficacy and develop responses to $P$. falciparum drug resistance across the GMS. Since 2000, the DVBD monitored drug efficacy from in vivo TES conducted in Thailand with mefloquine-artesunate combination therapy for $P$. falciparum malaria [13]. The aims of the subsequent multipronged Containment Project included increasing surveillance to provide information for the containment of artemisininresistant parasites [2]. By 2011, the Containment Project had supported surveillance of patients who tested positive after 3 days of treatment, and it had developed systematic processes for cross-border investigation and follow-up [13].

Surveillance to detect changes in P. falciparum susceptibility to deployed ACTs is critical for maintaining momentum towards malaria elimination. However, the decline in malaria incidence in Thailand means that it is more difficult to recruit the minimum patient sample size for TES. Given the constant threat of drug-resistant strains, a gap in surveillance where the efficacy of deployed drug regimens is essentially unknown would be unacceptable. Ineffective antimalarial therapy drives the spread of resistant parasites, so it is critical that drug efficacy is maintained and that regimens are switched promptly where necessary [14]. Also, monitoring the operational effectiveness of radical cure with chloroquine/primaquine is essential for diminishing the hypnozoite reservoir and reducing vivax malaria incidence and transmission.

Integrated drug efficacy surveillance (iDES) is a novel approach that incorporates drug resistance monitoring as part of routine case-based surveillance and response. iDES expanded the initiatives undertaken in the Containment Project, requiring that all malaria cases, symptomatic or asymptomatic, have a laboratory confirmed malaria diagnosis and receive treatment according to national guidelines, with parasitological follow-up to ensure parasite clearance. iDES aims to support evidence-based strategic policy development and operational decision making to realize malaria elimination while ensuring patient outcomes. This study examines the trends and status of malaria in Thailand, the implementation of iDES, initial measures of program performance, and the potential for further development. The contribution of iDES data to decision making in the context of malaria elimination is also discussed.

\section{Methods}

\section{iDES aims and implementation}

Thailand's malaria elimination strategy requires that all patients have malaria diagnosed by microscopy or rapid diagnostic test, receive supervised treatment in adherence to the national treatment guidelines, and are followed up four times to ensure cure [5, 15]. iDES supports these aims by monitoring adherence to treatment guidelines, tracking follow-up rates, and recording clinical and parasitological outcomes as part of routine case management [16]. From May 2017, the iDES protocol, updated from previous in vivo studies, was piloted in three provinces in northern Thailand (Chiang Mai, Chiang Rai, and Mae Hong Son), with

Page $3 / 22$ 
expansion to eight provinces in fiscal year 2017 (FY2017; i.e., 1 October 2017 to 31 September 2018) [16]. National rollout of the iDES protocol commenced in FY2018. The MIS was upgraded to allow data capture from iDES activities, and analytics and visualizations were developed to allow routine data interrogation [11]. The iDES system aims to routinely capture epidemiological and laboratory data from all patients diagnosed with malaria in Thailand as part of routine malaria care. The iDES protocol outlines aims, treatment, follow-up, data recording, and sample collection and submission [16]. The methods described here are consistent with ongoing iDES management and operational procedures.

\section{Treatment}

All treatment was provided free of charge in all health facilities responsible for malaria case management - malaria posts, border malaria posts, malaria clinics, health-promoting hospitals, and public and private hospitals [17]. Treatment was prescribed as per the Practice Guidelines for the Treatment of Patients with Malaria, Thailand, 2019 (Table 1) [15]. Artesunatemefloquine was replaced in 2015 with dihydroartemisinin-piperaquine as first-line therapy for $P$. falciparum malaria, with rollout in 2016. In FY2019, dihydroartemisinin-piperaquine was withdrawn from Sisaket and Ubon Ratchathani Provinces in north-east Thailand due to the high treatment failure rates observed from iDES and confirmed by another study [18]; it was replaced with pyronaridine-artesunate. Single-dose primaquine (30 mg) was required for $P$. falciparum cases to clear gametocytes [19]. Radical cure of $P$. vivax or $P$. ovale malaria was recommended to clear hypnozoites with chloroquine plus 14-day primaquine $(0.25 \mathrm{mg} / \mathrm{kg} /$ day). Note that primaquine was not given if the patient was pregnant, under 2 years of age, or glucose-6phosphate dehydrogenase (G6PD) deficient. In the case of confirmed G6PD deficiency, an 8-week treatment of weekly primaquine $(0.75 \mathrm{mg} / \mathrm{kg} /$ week $)$ instead of the 14 -day regimen was recommended for $P$. vivax/ovale radical cure. Any patient with a blood smear suspected to be $P$. knowlesi was referred to the district hospital for polymerase chain reaction (PCR)-based diagnosis and treatment. 


\section{P. falciparum}

First-line: DHA-PIP for 3 days + PQ single dose (30 mg or $0.5 \mathrm{mg}$ base/ $\mathrm{kg}$, started on day 1 pending patient's condition) (except for Sisaket and Ubon Ratchathani, where PY-AS for 3 days + PQ single dose was adopted in FY2019)

Second-line (ACT): 1. PY-AS 3 days + $P Q$ single dose; 2. AL for 3 days + $P Q$ single dose; 3 . AS-MQ for 3 days + $P Q$ single dose

Second-line (non-ACT): 1. Quinine + clindamycin-doxycycline-tetracycline for 7 days + PQ single dose; 2 . Atovaquoneproguanil for 3 days $+\mathrm{PQ}$ single dose

P. vivax or P. ovale

First-line: $\mathrm{CQ}$ for 3 days $+\mathrm{PQ}(0.25 \mathrm{mg}$ base/kg per day, started on day 3$)$ for 14 days

Second-line: DHA-PIP for 3 days + PQ for 14 days

P. malariae or P. knowlesi

First-line: CQ for 3 days

Second-line: DHA-PIP for 3 days

P. falciparum plus P. vivax or P. ovale

DHA-PIP for 3 days + PQ for 14 days

P. falciparum plus P. malariae or P. knowlesi

DHA-PIP for 3 days $+P Q$ single dose

\section{Severe malaria}

First-line: Artesunate injection within 24 h, followed by first-line/second-line regimen when tolerated + supportive care

Second-line: Quinine injection within first $24 \mathrm{~h}$ followed by first-line/second-line regimen when tolerated + supportive care

\section{Uncomplicated malaria in pregnancy 1 st trimester*}

Quinine + clindamycin for 7 days (P. falciparum or P. knowlesi)

CQ for 3 days (P. vivax, P. ovale, or P. malariae)

\section{Uncomplicated malaria in pregnancy 2nd or 3rd trimester*}

DHA-PIP for 3 days (P. falciparum or P. knowlesi)

CQ for 3 days (P. vivax, P. ovale, or P. malariae)

*Primaquine and doxycycline-tetracycline are contraindicated during pregnancy.

$A C T$, artemisinin-based combination therapy; DHA-PIP, dihydroartemisinin-piperaquine; $P Q$, primaquine; $P Y-A S$, pyronaridineartesunate; $\mathrm{AS}-\mathrm{MQ}$, artesunate-mefloquine; $\mathrm{AL}$, artemether-lumefantrine; $\mathrm{CQ}$, chloroquine.

\section{Follow-up}

Patients with falciparum malaria infection were followed up at days $3,7,28$, and 42 (from the day of diagnosis and prescribed treatment) and those with vivax malaria at days 14, 28, 60, and 90. For each follow-up visit, the clinical team noted the date, patient temperature, microscopy findings (species, parasite density, presence of gametocytes), and confirmation of outcome as recrudescence or re-infection. Patients were asked if they had been consuming the antimalarial drugs as prescribed, and the drug bag was requested for observation as a proxy of treatment compliance. For $P$. falciparum cases, the PCR genotyping results could be added later to the patient record (see below).

\section{Data recording}


The officer from the laboratory reference center was responsible for data recording, either at a reference laboratory (DVBD) or a regional laboratory (Department of Disease Control). The reporting requirements were consistent with those used in TES to enable comparison with historical data and between countries [20]. The patient's name, age, sex, weight, height, temperature, address, and residency background (i.e., resident Thai, long-term migrant [ $\geq 6$ months residency], or short-term migrant $[<6$ months residency]), were recorded, as well as the date that blood samples were taken and the date on which the person visited the clinic. Malaria species, parasite density, and the presence of gametocytes were assessed using standard methods by trained microscopists and reported [20,21]. The prescribed antimalarial drug and number of tablets/capsules were noted. All of these data were captured on the Malaria Case Follow-Up form and stored in the MIS (Fig. 1).

\section{Sample collection and submission}

Blood spots were collected onto filter paper from all patients at first consultation and at follow-up visits. Samples were sent to regional and national laboratories as part of the microscopy quality assurance system. In the case of $P$. falciparum, PCR genotyping was used to compare samples from the initial and recurrent infection to differentiate recrudescence from reinfection, according to published methods [22]. For P. falciparum cases, blood samples were also collected for analysis of Kelch 13 gene mutations associated with artemisinin resistance and Pfmdr1 gene copy number [23, 24]. Data on molecular markers will be reported separately.

\section{Outcomes and statistical analysis}

The primary outcome of the analysis was the crude drug efficacy rate among patients who had microscopically confirmed malaria with an identified Plasmodium species at baseline, received first-line treatment, and had at least one post-baseline follow-up visit. Treatment failure was defined as microscopically confirmed parasite reappearance within 42 days of first-line therapy for $P$. falciparum (dihydroartemisinin-piperaquine + primaquine) and within 28 days of first-line therapy for $P$. vivax (chloroquine + primaquine). Additionally, P. vivax recurrence rate (relapse/re-infection) was determined at day 60 and day 90 . Crude cure rates were reported without PCR adjustment, as PCR data were sparse. Drug efficacy was estimated using KaplanMeier methods. Patients were censored if they had a re-infection with a different species from their initial infection or at their final treatment outcome assessment. In order to assess programmatic efficacy, all re-treatments with first-line therapy following failure were considered to be new malaria episodes, and the patients were requested to report for another four follow-up visits to ensure complete cure. All other outcomes were reported using descriptive statistics. Data were downloaded from the MIS on 16 November 2020. Statistical analysis used Stata (version 16) and GraphPad Prism (version 9.0.0).

\section{Results}

\section{Malaria trends}

Between FY2013 and FY2020, there was an overall 88.1\% decrease in malaria incidence from 37,741 to 4,474 cases (Fig. 2). There was a sustained decline in the incidence of both $P$. falciparum and $P$. vivax malaria, but with a marked change in the relative proportions of infections caused by these two parasites; in FY2013, 43.7\% $(16,494 / 37,741)$ of infections were caused by P. falciparum, whereas in FY2020, this figure had declined to $5.7 \%(257 / 4,474)$ (Fig. 2). Over the same period, malaria mortality declined from 47 to 0 deaths (Fig. 2). The number of active malaria foci (defined as subvillages with ongoing malaria transmission) also contracted, from 2,387 in FY2013 to 605 in FY2020, representing a 74.7\% decrease (Fig. 2).

\section{Current malaria situation}

From data recorded in the MIS, in FY2020 there were 4,474 malaria cases, representing a case incidence rate of $0.067 / 1,000$ population, against the FY2020 target for malaria elimination of $0.22 / 1,000$ population [5]. P. falciparum accounted for $5.7 \%$ $(257 / 4,474)$ of cases and $P$. vivax for $91.6 \%(4,099 / 4,474)$ (Fig. 2). The remaining cases were classified as $P$. malariae $(\mathrm{n}=53)$, $P$. knowlesi $(\mathrm{n}=11)$, or mixed $(\mathrm{n}=18)$ (Fig. 2). Thirty-six cases were unknown, with no $P$. ovale recorded. Malaria incidence was concentrated along the western border that Thailand shares with Myanmar, particularly Tak and Kanchanaburi Provinces; in the south of the country bordering Malaysia in Yala Province; and in the northeast bordering Cambodia in Sisaket and Ubon

Page 6/22 
Ratchathani Provinces (Fig. 3). The peak malaria transmission season occurred between April and September, with a lower peak in October (Fig. 3).

Most malaria infections occurred in individuals who were at least 15 years old $(70.2 \%[3,142 / 4,474])$, and around two-thirds of infections occurred in males (65.9\% [2,950/4,474]) (Fig. 4). Most infections were acquired in the same household (56.8\% [2,543/4,474]), but around a fifth of cases were imported (21.3\% [954/4,474]) (Fig. 4). Thai residents accounted for 71.3\% $(3,190 / 4,474)$ of cases, with the remaining cases split between long-term migrants $(14.9 \%[666 / 4,474])$ and short-term migrants $(13.8 \%[618 / 4,474])$ (Fig. 4).

\section{Follow-up rates and adherence to national treatment guidelines}

The proportion of patients with outcomes recorded at day 42 for $P$. falciparum malaria and for 28 days for $P$. vivax malaria has been increasing each year since the national launch of iDES (Fig. 5A). In FY2020, 61.5\% (158/257) of $P$. falciparum cases had a treatment outcome recorded on day 42 , and $57.2 \%(2,344 / 4,099)$ of $P$. vivax cases had a day 28 outcome recorded (Fig. 5A). For P. falciparum malaria, day 42 follow-up rates were highest for long-term migrants $(80.6 \%$ [29/36]) and Thai residents $(64.8 \%$ [127/196]), but lower for short-term migrants (8.0\% [2/25]) (Fig. 5B). A similar pattern was observed for $P$. vivax malaria, with $77.6 \%(471 / 607)$ of long-term migrants, $62.7 \%(1,821 / 2,905)$ of Thai residents, and $8.9 \%(52 / 587)$ of short-term migrants followed up at day 28 (Fig. 5B).

The proportion of evaluable patients who received antimalarial therapy aligned with national treatment guidelines improved over the iDES period, from $70.7 \%(4,212 / 5,956)$ in FY2018, to $77.2 \%(4,103 / 5,315)$ in FY2019 and 84.2\% $(3,549 / 4,215)$ in FY2020.

\section{Antimalarial drug efficacy}

Antimalarial drug efficacy was assessed for those patients who received at least one dose of first-line treatment and had at least one follow-up visit. Recurrences occurred in different provinces in different years, though Sisaket, Tak, and Yala had $P$. vivax recurrences in all three iDES years (Table 2). P. vivax recurrences also varied by follow-up day, with the majority of positive tests occurring on days 60 and 90 (Table 3).

Only provinces with malaria recurrences are shown. Recurrences are shown for patients who had at least one follow-up visit for P. falciparum malaria up to and including day 42 and received dihydroartemisinin-piperaquine plus primaquine; and for patients who had at least one follow-up visit for P. vivax malaria up to and including day 90 and received chloroquine plus primaquine. 
Table 2. Summary of malaria recurrences by year, species and province

\begin{tabular}{|c|c|c|c|}
\hline \multirow[t]{2}{*}{ Species and province } & \multicolumn{3}{|c|}{ Recurrences, n/N (\%) } \\
\hline & FY2018 & FY2019 & FY2020 \\
\hline P. falciparum overall & $13 / 249(5.2)$ & 14/379 (3.7) & 3/167 (1.8) \\
\hline Chanthaburi & - & - & 2/6 (33.3) \\
\hline Kamphaeng Phet & - & $1 / 1(100)$ & - \\
\hline Phangnga & $1 / 2(50.0)$ & - & - \\
\hline Sakaeo & - & 1/3 (33.3) & - \\
\hline Sisaket & 8/59 (13.6) & $10 / 49(20.4)$ & - \\
\hline Surat Thani & 2/33 (6.1) & - & $1 / 15(6.7)$ \\
\hline Surin & $1 / 4(25.0)$ & $1 / 7(14.3)$ & - \\
\hline Tak & - & $1 / 52(1.9)$ & - \\
\hline Ubon Ratchathani & $1 / 10(10.0)$ & - & - \\
\hline P. vivax overall & 66/2048 (3.2) & $58 / 2206(2.6)$ & $52 / 2448(2.1)$ \\
\hline Chachoengsao & $2 / 42(4.8)$ & - & - \\
\hline Chon Buri & $1 / 6(16.7)$ & - & - \\
\hline Kanchanaburi & 1/143 (0.7) & $3 / 235$ (1.3) & - \\
\hline Mae Hong Son & $3 / 184(1.6)$ & 6/199 (3.0) & - \\
\hline Nakhon Ratchasima & - & $2 / 2(100)$ & $1 / 19(5.3)$ \\
\hline Phetchaburi & - & $2 / 79(2.5)$ & 4/124 (3.2) \\
\hline Phitsanulok & - & - & $2 / 26(7.7)$ \\
\hline Prachin Buri & $1 / 22(4.5)$ & - & - \\
\hline Prachuap Khiri Khan & - & $2 / 65(3.1)$ & 6/110 (5.5) \\
\hline Ratchaburi & - & $7 / 117(6.0)$ & $11 / 182(6.0)$ \\
\hline Sisaket & $36 / 200$ (18.0) & 24/198 (12.1) & $5 / 41(12.2)$ \\
\hline Tak & 20/385 (5.2) & 8/398 (2.0) & 21/703 (3.0) \\
\hline Yala & 2/506 (0.4) & 4/523 (0.8) & 2/559 (0.4) \\
\hline
\end{tabular}

For $P$. falciparum malaria, day 42 efficacy (Kaplan-Meier) was $92.4 \%(95 \% \mathrm{Cl} 87.1,95.6)$ in $\mathrm{FY} 2018,93.3 \%(95 \% \mathrm{Cl} 88.8,96.0)$ in FY2019, and 98.0\% $(95 \% \mathrm{Cl} 93.9,99.4)$ in FY2020 (Fig. 6). Most falciparum recurrences occurred in Sisaket Province, with day 42 efficacy rates of $75.9 \%(95 \% \mathrm{Cl} 56.0,87.8)$ in FY2018 and 49.4\% (95\% Cl 24.8, 70.0) in FY2019 (Fig. 6). In FY2018, all eight treatment failures in Sisaket occurred in different individuals. In FY2019, ten treatment failures occurred in six patients, three of whom received repeated treatment with dihydroartemisinin-piperaquine rather than second-line therapy. However, even when only the first treatment episode was considered for these patients, the day 42 efficacy rate in Sisaket in FY2019 was 61.5\% 
(95\% Cl 31.2, 81.7). Data were triangulated with PCR results from blood spots, which were available for six of the FY2018 patients and two of the FY2019 patients. In February 2019, pyronaridine-artesunate replaced dihydroartemisinin-piperaquine as first-line therapy against uncomplicated falciparum malaria in Sisaket and neighboring Ubon Ratchathani, with rollout in FY2020. In FY2020, there were only three $P$. falciparum cases in Sisaket and Ubon Ratchathani. Two cases received pyronaridine-artesunate, with no recurrence. One received dihydroartemisinin-piperaquine; after parasite reappearance at day 60, the case was successfully treated with pyronaridine-artesunate.

For $P$. vivax malaria, chloroquine/primaquine day 28 efficacy was at least $98 \%$ across all iDES years and day 60 efficacy was > 95\% (Fig. 7). Day 90 efficacy was 94.8\% (95\%Cl 93.4, 95.9) in FY2018, but improved to 97.1\% (96.2, 88.7) in 2020 (Fig. 7).

Sisaket Province proportionally had the greatest effect on the overall failure rate. Day 28 efficacy in Sisaket was $85.2 \%(95 \% \mathrm{Cl}$ $77.3,90.5)$ in FY2018, but improved to $95.0 \%(95 \% \mathrm{Cl} 90.0,97.5)$ in FY2019, and 100\% in FY2020 (Fig. 7). However, day 60 and day 90 efficacy in Sisaket remained sub-optimal, with both at $61.7 \%(95 \% \mathrm{Cl} 50.8,71.0)$ in $\mathrm{FY} 2018,76.4 \%(95 \% \mathrm{Cl} 66.4,83.8)$ in FY2019, and 75.0\% (95\% Cl 50.0, 88.7) in FY2020 (Fig. 7).

Table 3. Summary of $P$. vivax malaria recurrences by year and follow-up day

Recurrences are shown for patients with at least one follow-up visit for $P$. vivax up to and including day 90 who received chloroquine plus primaquine.

\begin{tabular}{|llll|}
\hline Species and follow-up day & \multicolumn{2}{l|}{ Recurrences, $\mathrm{n} / \mathrm{N}(\%)$} \\
\cline { 2 - 4 } & $\mathrm{FY2018}$ & $\mathrm{FY2019}$ & $\mathrm{FY2020}$ \\
\hline P. vivax overall & $66 / 2048(3.2)$ & $58 / 2206(2.6)$ & $52 / 2448(2.1)$ \\
\hline Day 14 & $1(1.5)$ & $7(12.1)$ & $2(3.8)$ \\
\hline Day 21 & $2(3.0)$ & $1(1.7)$ & 0 \\
\hline Day 28 & $20(30.3)$ & $10(17.2)$ & 0 \\
\hline Day 35 & 0 & $1(1.7)$ & 0 \\
\hline Day 42 & 0 & 0 & $1(1.9)$ \\
\hline Day 60 & $36(54.5)$ & $31(53.4)$ & $21(40.4)$ \\
\hline Day 90 & $7(10.6)$ & $8(13.8)$ & $28(53.8)$ \\
\hline
\end{tabular}

\section{Box 1. Intensified iDES to support treatment policy change}


$100 \%$ adherence to the national treatment guidelines

No stock-outs of drugs

Additional training for treatment providers to ensure daily supervised drug intake

Target to achieve $>90 \%$ of follow-up days

iDES standard operating procedures are followed with increased frequency of monitoring

Adequate patient support for follow-up visits (providing transport, etc.)

Quality control on all microscopy slides

Collection of all day 0 dried blood spots and at recurrence for PCR and molecular markers

Follow-up of all treatment failures

Integration of laboratory data and the results of molecular markers to the online system

\section{Discussion}

Thailand has made significant progress towards malaria elimination, surpassing its 2020 milestone reductions for both malaria incidence and mortality [8]. In particular, the incidence of falciparum malaria has declined steeply. Although $P$. vivax malaria incidence has also fallen, this parasite has been more refractory to malaria control interventions. The highest malaria incidence rates are along the Thai-Myanmar border, a rural and densely forested region with a highly mobile population [6]. Also, many areas remain receptive to malaria, and re-establishment of transmission continues to be a significant risk across most of the country [25].

It was clear that as malaria incidence declined in Thailand, TES would no longer be feasible in many provinces. In low transmission settings, TES usually requires at least 50 patients, ideally recruited within a single malaria season [20]. Given the high risk of malaria outbreaks potentially involving $P$. falciparum resistant to first-line antimalarial drugs, continued drug efficacy surveillance was imperative. Also, as the importance of $P$. vivax increased, ensuring effective implementation of radical cure was necessary to prevent relapses and further transmission.

The reorganization of the health system to target malaria elimination offered an opportunity to integrate drug efficacy surveillance. In order to move from TES to iDES, it was necessary for Thailand to have a strong case-based surveillance system and the capacity for universal and supervised antimalarial treatment. The MIS was introduced countrywide in 2012 to improve case reporting, analysis, and response planning and to monitor implementation of malaria elimination activities. Public health officials were able to access automated information presentations and reports which could be used to evaluate malaria control efforts and respond rapidly to malaria outbreaks. Retroactive adaptation of the MIS to incorporate the iDES module was complex and has required continued refinement of the platform to adapt to changing epidemiology and corresponding data needs. The DVBD can drill down into the data in almost real time, thereby enabling limited resources to be targeted to the provinces, villages, or health facilities most in need of support to improve iDES compliance. The DVBD and its partners are working to further improve data visualizations to facilitate the data analyses most needed by subnational officers, such as health care workers interested in patient follow-up and treatment outcomes.

iDES is only appropriate in an elimination setting where most cases can be followed until resolution. For countries that have not yet reached the elimination phase, TES remains the most appropriate drug efficacy surveillance method [17]. For iDES to have an impact on policy, other aspects of the health system need to be aligned for delivering different antimalarial drugs to different 
regions. For example, purchasing and supply logistics, laboratory capacity and coordination, health financing, communication with prescribers, community outreach, and patient education. Thus, iDES cannot just be appended to an information system, but instead must be fully integrated into all health system processes.

Rapid diagnostic tests for malaria have been an invaluable tool in areas of high-to-moderate transmission to identify cases and direct appropriate therapy. However, these tests cannot verify parasite clearance, and so drug efficacy cannot be determined. iDES requires microscopic malaria diagnosis, yet maintaining microscopy skills in an elimination setting is challenging as laboratories may see very few cases in some regions. To address this issue, the Thailand Ministry of Public Health organizes regular quality control microscopy testing and training, including competency assessments and training of trainers, with assistance from partner agencies to develop standard operating procedures [26]. With sustained support from external funding partners, Thailand has invested in a strong cadre of trained microscopists stationed throughout the country, and 33 professionals hold current expert certification from the WHO. However, other countries in the GMS and elsewhere may need to consider how to build these skills as malaria burden reduces, and as an iDES system becomes the recommended program for case-based surveillance and follow-up.

Despite being included in the iDES protocol, the collection of dried blood spots for PCR analysis of $P$. falciparum has been sporadic. Thus, only crude efficacy rates are reported here, but in a country aiming for elimination, it is expected that all recurrences are recrudescences. The collection of samples for molecular resistance markers has also been sub-optimal while subnational officers gain new skills on a highly sensitive process. Although trends in antimalarial efficacy can still be evaluated, further action is needed to improve the collection of samples, with additional training for health workers and laboratory staff. The DVBD national laboratory is expending substantial time and energy to improve biomarker collection in ways that will enhance iDES analyses. As the case incidence of $P$. falciparum declines, it becomes more important that all potential data are collected and analyzed, and the evaluation of molecular markers becomes essential. The DVBD anticipates enhanced molecular surveillance and streamlined processes to triangulate clinical and laboratory data as a National Reference Laboratory database is developed. The delay between reporting clinical outcome and molecular markers of resistance is likely to contract as resources are developed and when data management is harmonized.

Follow-up rates for iDES have been increasing steadily since its introduction, supported by a network of village health volunteers and through community education. However, patients are still being lost between treatment initiation and the first follow-up visit. High burden provinces in western Thailand (Fig. 3) have lower follow-up rates, as does the crucial Sisaket Province. Also, follow-up rates among short-term migrants have been consistently low, largely because maintaining contact with this population is challenging. There is the potential to incorporate mobile health data (mHealth) within iDES, which could expand the coverage of malaria follow-up services to the household and individual levels via patients' mobile devices [27]. This intervention could be a route to boost follow-up rates in the most difficult-to-reach patients.

Complementary research on challenges to maximizing iDES follow-up rates would support a comprehensive plan to improve routine patient care and treatment outcomes. Identifying potential bottlenecks - such as patient resistance or forgetfulness, provider lack of knowledge, poor adherence, or issues with recording and reporting - would be a helpful step in developing a plan to fortify the iDES system. Improving patient follow-up would also support a more complete iDES dataset and provide sufficient data for tracking individual patient outcomes. A positive sign is that in FY2020, malaria case follow-up and iDES showed resilience to disruption caused by the novel coronavirus disease (COVID-19) epidemic, given that both follow-up rates and data capture improved. Historic data from Thailand's earlier follow-up programs also suggest that high rates can be achieved [13]. Thus, the existing system is robust and can support further development.

Adherence to the national treatment guidelines is necessary to optimize patient outcomes and to support malaria elimination. Although adherence rates have been improving, further progress will require additional resources. For example, Thailand's village health volunteers are key in accessing remote locations and engaging with patients on a personal level, and additional training of these health workers is planned in FY2021, alongside iDES capacity building. The aim is that all patients will receive the recommended antimalarial therapy, with complete daily treatment supervision and follow-up. 
Although follow-up rates and treatment adherence are not perfect in terms of ensuring individual case outcome, data penetration has been sufficient to enable policy decisions on antimalarial drug treatment at the provincial level. The identification of an unacceptably high clinical failure rate with dihydroartemisinin-piperaquine against $P$. falciparum in the Cambodian border areas enabled first-line therapy to be switched to pyronaridine-artesunate for two provinces. Pyronaridineartesunate has been shown to retain high efficacy in regions in the GMS where multidrug-resistant $P$. falciparum parasites are prevalent [28-31]. A TES study normally would be conducted to support a drug treatment policy change. However, pyronaridineartesunate efficacy in Sisaket and Ubon Ratchathani will be monitored in FY2021 through 'intensified iDES' (Box 1), which aims to optimize data gathering, given the anticipated low number of $P$. falciparum cases. The capacity to address drug resistance at this level allows containment of resistant parasites, with the continued potential for local elimination. However, in these border regions, cross-collaboration between countries may be required to ensure patient outcomes. In Sisaket, iDES also identified issues related to re-treatment with first-line therapy following failure, leading to subsequent treatment failure. These second treatment failures are programmatic and may result from stock-outs of second-line antimalarial therapies, patients presenting at different clinics, or patient or prescriber choice. Being able to find and interrogate these cases would allow interventions to be targeted at the causes of non-adherence to treatment guidelines in specific locations. Given the low and declining malaria incidence in Thailand, without iDES it is unlikely that a pattern of treatment failure would be detected in time to avert an outbreak of drug-resistant $P$. falciparum. There is also evidence that iDES can support appropriate management of imported malaria as part of a comprehensive prevention of reintroduction program [32]. Maintaining iDES may be crucial for Thailand as neighboring countries in the GMS strive for elimination in the coming years.

iDES data also underline the importance of $P$. vivax malaria elimination. Although generally high efficacy rates were observed, a disparity was evident in Sisaket versus other provinces. It is not possible to determine whether the treatment failures in Sisaket were caused by recrudescence (chloroquine failure), relapse (primaquine failure), or re-infection. The high day 28 failure rate in Sisaket in FY2018 suggests that chloroquine therapy may have been sub-optimal, though day 28 efficacy was $100 \%$ in FY2020. Mutations associated with chloroquine resistance have been detected in P. vivax isolates from Thailand [9]. Notably, Sisaket borders Cambodia, where chloroquine was abandoned in 2012 because of parasite resistance, being replaced first by dihydroartemisinin-piperaquine, and then by mefloquine-artesunate in 2017. There is some evidence of increasing chloroquine susceptibility in Cambodian clinical isolates [33], which could explain the improved day 28 efficacy observed in Sisaket between FY2018 and FY2020. However, day 90 efficacy has remained unacceptably low in Sisaket. Although primaquine treatment should be fully supervised, drug consumption cannot be verified and suboptimal primaquine adherence cannot be excluded as a cause. Cytochrome 2D6 polymorphisms can affect primaquine efficacy [34], but this was not investigated for these patients. There may also be social factors in this particular region that predispose the population to reinfection, despite declining case numbers. Investigations and discussions on the appropriate response to $P$. vivax malaria in Sisaket are ongoing in FY2021, and the findings may also affect management of $P$. vivax malaria elsewhere in Thailand.

iDES has provided operationally relevant data on drug efficacy; however, the DVBD is working to address remaining implementation challenges. For example, the attrition rate during the follow-up period was high, especially at the start of iDES and particularly with the long 90-day window required for $P$. vivax cases. Supervising drug consumption, even in a low prevalence setting, requires extensive resources. While village health volunteers play a major role in this realm, observing the drug pack as a proxy for consumption, coverage may vary. Thus, iDES cannot establish with certainty the efficacy of primaquine in attaining radical cure in $P$. vivax cases. Even in a country with high microscopy and laboratory capacity like Thailand, appropriate storage and rapid processing of iDES samples remains a challenge, hindering quality assurance. Finally, the DVBD is improving data management to facilitate integrated patient files and triangulation of epidemiological and laboratory data for iDES analyses.

\section{Conclusion}

The investment in malaria elimination in Thailand is considerable, but the potential benefits are even greater [35]. As countries approach elimination, it is likely that the most resistant parasites will be those that remain in circulation [14]. Ineffectual therapy promotes the spread of drug-resistant parasites, and risks malaria resurgence and the re-establishment of transmission. Thus,

Page $12 / 22$ 
as it becomes more difficult to conduct drug resistance surveillance activities, it also becomes increasingly important to understand which drugs retain efficacy and whether treatment policies should be amended. As an approach for malaria elimination, iDES is designed to encompass all malaria cases, whether imported or indigenous; to encourage treatment compliance; and to follow patients until they are clinically cured and confirmed as parasite free. Thailand's experience with iDES offers a pragmatic model for malaria-eliminating countries where TES is no longer feasible. iDES can be a useful approach to target malaria elimination by ensuring that all malaria patients receive appropriate treatment and are ultimately cured of malaria.

\section{Abbreviations}

ACT artemisinin-based combination therapy

$\mathrm{AL}$ artemether-lumefantrine

AS-MQ artesunate-mefloquine

COVID-19 Coronavirus disease of 2019

CQ chloroquine

DHA-PIP dihydroartemisinin-piperaquine

DVBD Division of Vector Borne Diseases

FY fiscal year

G6PD glucose-6-phosphate dehydrogenase

GMS Greater Mekong Subregion

iDES integrated drug efficacy surveillance

MIS Malaria Information System

PCR polymerase chain reaction

$\mathrm{PQ}$ primaquine

PY-AS pyronaridine-artesunate

TES therapeutic efficacy studies

USAID United States Agency for International Development

WHO World Health Organization

\section{Declarations}

\section{Ethics approval and consent to participate}

Not applicable.

\section{Consent for publication}




\section{Availability of data and material}

All relevant data are provided in the manuscript or are available from the website of the Department of Disease Control, Ministry of Public Health Thailand: https//malaria.ddc.moph.go.th.

\section{Competing interests}

PS, AS, NK, TT, SK, RS, CL, and DA are employees of the Division of Vector Borne Diseases, Ministry of Public Health, Thailand. JAS and SN are employees of RTI International, Thailand. DG, PR, and MDB are staff members of the World Health Organization; the authors alone are responsible for the views expressed in this publication that do not necessarily represent the decisions, policy, or views of the World Health Organization. NP and DS are employees of the Regional Development Mission for Asia, United States Agency for International Development, Thailand. The authors have no further conflicts of interest to declare.

\section{Funding}

This study was made possible by the generous support of the American people through the U.S. President's Malaria Initiative and United States Agency for International Development (USAID), under the terms of Cooperative Agreement AID-486-LA-1500002 for Inform Asia: USAID's Health Research Program. iDES implementation was also supported by the Thailand Division of Vector Borne Diseases (DVBD), the U.S. President's Malaria Initiative (PMI), the World Health Organization, and the Global Fund to Fight AIDS, Tuberculosis, and Malaria.

\section{Authors' contributions}

PS, AS, NK, DG, MDB, and PR were instrumental in designing and establishing the iDES system for Thailand. TT and SK led routine monitoring of iDES results. PS, AS, DG, and JAS contributed to defining the scope and content of the review; JAS assembled and analyzed the data. All authors reviewed the data and results, critically reviewed the manuscript, and approved the final version of the manuscript for publication.

\section{Acknowledgements}

The authors recognize the contribution of the community volunteers and public health staff, especially from the Division of Vector Borne Diseases, Department of Disease Control, Ministry of Public Health, Thailand. Naomi Richardson developed the first draft of this paper, provided editorial and graphic services, cross-checked all data and analyses, and was funded by Inform Asia: USAID's Health Research Program.

\section{Disclaimer}

Any opinions expressed in this supplement are those of the authors and do not necessarily reflect the views of the PMI, or the United States Agency for International Development. DG, PR, and MDB are staff members of the World Health Organization (WHO) and are responsible for the views expressed in this publication, which do not necessarily reflect the decisions or policies of the WHO.

\section{References}


1. Imwong M, Dhorda M, Myo Tun K, Thu AM, Phyo AP, Proux S, et al. Molecular epidemiology of resistance to antimalarial drugs in the Greater Mekong subregion: an observational study. Lancet Infect Dis. 2020;20:1470-80.

2. Khamsiriwatchara A, Sudathip P, Sawang S, Vijakadge S, Potithavoranan T, Sangvichean A, et al. Artemisinin resistance containment project in Thailand. (I): Implementation of electronic-based malaria information system for early case detection and individual case management in provinces along the Thai-Cambodian border. Malar J. 2012;11:247.

3. WHO Regional Office for the Western Pacific. Strategy for malaria elimination in the Greater Mekong Subregion: 2015-2030. World Health Organization Regional Office for the Western Pacific. 2015. https://apps.who.int/iris/handle/10665/208203. Accessed 18 February 2021.

4. Bureau of Vector Borne Diseases, Department of Disease Control, Ministry of Public Health Thailand. Guide to malaria elimination for Thailand's local administrative organizations and the health network. Ministry of Public Health Thailand. 2019.

http://malaria.ddc.moph.go.th/downloadfiles/Guide\%20to\%20Malaria\%20Elimination\%20for\%20Thailand\%20LAO_EN.pdf. Accessed 9 October 2020.

5. Bureau of Vector Borne Diseases, Department of Disease Control, Ministry of Public Health Thailand. National malaria elimination strategy for Thailand 2017-2026. Nonthaburi: Ministry of Public Health Thailand; 2016.

6. Sudathip P, Kitchakarn S, Thimasarn K, Gopinath D, Naing T, Sajjad O, et al. The evolution of the malaria clinic: the cornerstone of malaria elimination in Thailand. Trop Med Infect Dis. 2019;4:143.

7. USAID. US President's malaria initiative: Thailand, Lao PDR and regional abbreviated malaria operation plan FY 2019. United States Agency for International Development. 2019. https://www.pmi.gov/docs/default-source/default-documentlibrary/malaria-operational-plans/fy19/fy-2019-thailand-abbreviated-malaria-operational-plan.pdf?sfvrsn=5. Accessed 9 October 2020.

8. WHO. World malaria report. World Health Organization. 2020. https://www.who.int/teams/global-malariaprogramme/reports/world-malaria-report-2020. Accessed 13 December 2020.

9. Tantiamornkul K, Pumpaibool T, Piriyapongsa J, Culleton R, Lek-Uthai U. The prevalence of molecular markers of drug resistance in Plasmodium vivax from the border regions of Thailand in 2008 and 2014. Int J Parasitol Drugs Drug Resist. 2018;8:229-37.

10. Olliaro PL, Barnwell JW, Barry A, Mendis K, Mueller I, Reeder JC, et al. Implications of Plasmodium vivax biology for control, elimination, and research. Am J Trop Med Hyg. 2016;95:4-14.

11. Division of Vector Borne Diseases, Department of Disease Control, Ministry of Public Health Thailand. Malaria Online: the digital surveillance system for Thailand malaria elimination. Ministry of Public Health Thailand. 2020.

https://publicadministration.un.org/unpsa/Portals/0/UNPSA_Submitted_Docs/2019/3fe4c1ba-e00b-4250-8816f513c3b209c6/2020\%20UNPSA_Malaria\%20online_full\%20report_27112019_111848_f62725d9-bd75-4846-a44c8524838f4e87.pdf?ver=1441-03-30-111848-927. Accessed 10 October 2020.

12. USAID. US President's malaria initiative: Thailand, Lao PDR, and regional malaria operation plan FY 2018. United States Agency for International Development. 2018. https://www.pmi.gov/docs/default-source/default-document-library/malariaoperational-plans/fy-2018/fy-2018-thailand-regional-malaria-operational-plan.pdf?sfvrsn=8. Accessed 9 October 2020.

13. Satimai W, Sudathip P, Vijaykadga S, Khamsiriwatchara A, Sawang S, Potithavoranan T, et al. Artemisinin resistance containment project in Thailand. II: Responses to mefloquine-artesunate combination therapy among falciparum malaria patients in provinces bordering Cambodia. Malar J. 2012;11:300.

14. Maude RJ, Pontavornpinyo W, Saralamba S, Aguas R, Yeung S, Dondorp AM, et al. The last man standing is the most resistant: eliminating artemisinin-resistant malaria in Cambodia. Malar J. 2009;8:31.

15. Ministry of Public Health Thailand. Practice guidelines for the treatment of patients with malaria, Thailand 2019. Nonthaburi: Ministry of Public Health Thailand; 2019.

16. Division of Vector Borne Diseases, Department of Disease Control, Ministry of Public Health Thailand: Standard operating procedures (SOP) for malaria case follow up in Thailand. Nonthaburi: Ministry of Public Health Thailand; 2019. 
17. WHO Regional Office for the Western Pacific. Fifth Meeting of the Greater Mekong Subregion (GMS) Therapeutic Efficacy Studies (TES) Network, Ho Chi Minh City, Viet Nam, 28-29 September 2017: meeting report. World Health Organization Regional Office for the Western Pacific. 2017. https://apps.who.int/iris/handle/10665/260008. Accessed 10 October 2020.

18. van der Pluijm RW, Imwong M, Chau NH, Hoa NT, Thuy-Nhien NT, Thanh NV, et al. Determinants of dihydroartemisininpiperaquine treatment failure in Plasmodium falciparum malaria in Cambodia, Thailand, and Vietnam: a prospective clinical, pharmacological, and genetic study. Lancet Infect Dis. 2019;19:952-61.

19. Stepniewska K, Humphreys GS, Gonçalves BP, Craig E, Gosling R, Guerin PJ, et al. Efficacy of single-dose primaquine with artemisinin combination therapy on Plasmodium falciparum gametocytes and transmission: an individual patient metaanalysis. J Infect Dis. 2020;20:943-52.

20. WHO. Methods for surveillance of antimalarial drug efficacy. World Health Organization. 2009. https://www.who.int/malaria/publications/atoz/9789241597531/en/. Accessed 13 December 2020.

21. WHO/TDR. Microscopy for the detection, identification and quantification of malaria parasites on stained thick and thin blood films in research settings. World Health Organization Special Programme for Research and Training in Tropical Diseases. 2015. https://www.who.int/tdr/publications/microscopy_detec_ident_quantif/en/. Accessed 10 October 2020.

22. WHO. Methods and techniques for clinical trials on antimalarial drug efficacy: genotyping to identify parasite populations. World Health Organization. 2008. http://www.who.int/malaria/publications/atoz/9789241596305/en/. Accessed 13 December 2020.

23. Institut Pasteur du Cambodge. PCR and sequencing for genotyping of candidate Plasmodium falciparum artemisinin resistance SNPs in the Kelch 13 gene v1.0. Institut Pasteur du Cambodge. 2013. https://www.wwarn.org/toolsresources/procedures/pcr-and-sequencing-genotyping-candidate-plasmodium-falciparum-artemisinin. Accessed 20 February 2021.

24. WWARN. Copy number estimation of $P$. falciparum pfmdr1 v1.1. WorldWide Antimalarial Resistance Network. 2011. https://www.wwarn.org/sites/default/files/attachments/procedures/MOL05_CopyEstimationPfalciparum_pfmdr1.pdf. Accessed 9 October 2020.

25. Sudathip P, Kitchakarn S, Shah J, Bisanzio D, Young F, Gopinath D, et al. A foci cohort analysis to monitor successful and persistent foci under Thailand's malaria elimination strategy. Malar J. 2021;20:118.

26. USAID. US President's malaria initiative Thailand, Lao PDR and regional malaria operational plan FY 2020. United States Agency for International Development. 2020. https://www.pmi.gov/docs/default-source/default-document-library/malariaoperational-plans/fy20/fy-2020-thailand-malaria-operational-plan.pdf?sfvrsn=6. Accessed 10 October 2020.

27. WHO. mHealth: new horizons for health through mobile technologies. World Health Organization. 2011. https://www.who.int/goe/publications/goe_mhealth_web.pdf. Accessed 27 October 2020.

28. Quang Bui P, Hong Huynh Q, Thanh Tran D, Thanh Le D, Quang Nguyen T, Van Truong H, et al. Pyronaridine-artesunate efficacy and safety in uncomplicated Plasmodium falciparum malaria in areas of artemisinin-resistant falciparum in Viet Nam (2017-2018). Clin Infect Dis. 2020;70:2187-95.

29. Leang R, Mairet-Khedim M, Chea H, Huy R, Khim N, Mey Bouth D, et al. Efficacy and safety of pyronaridine-artesunate plus single-dose primaquine for treatment of uncomplicated Plasmodium falciparum malaria in eastern Cambodia. Antimicrob Agents Chemother. 2019;63:e02242-18.

30. Leang R, Khim N, Chea H, Huy R, Mairet-Khedim M, Mey Bouth D, et al. Efficacy and safety of pyronaridine-artesunate plus single-dose primaquine for the treatment of malaria in western Cambodia. Antimicrob Agents Chemother. 2019;63:e0127319.

31. Han KT, Lin K, Han ZY, Myint MK, Aye KH, Thi A, et al. Efficacy and safety of pyronaridine-artesunate for the treatment of uncomplicated Plasmodium falciparum and Plasmodium vivax malaria in Myanmar. Am J Trop Med Hyg. 2020;103:108893.

32. Dharmawardena P, Rodrigo C, Mendis K, de AWGW, Premaratne R, Ringwald P, et al. Response of imported malaria patients to antimalarial medicines in Sri Lanka following malaria elimination. PLoS One. 2017;12:e0188613. 
33. Roesch C, Mairet-Khedim M, Kim S, Lek D, Popovici J, Witkowski B. Impact of the first-line treatment shift from dihydroartemisinin/piperaquine to artesunate/mefloquine on Plasmodium vivax drug susceptibility in Cambodia. J Antimicrob Chemother. 2020;75:1766-71.

34. Baird JK, Louisa M, Noviyanti R, Ekawati L, Elyazar I, Subekti D, et al. Association of impaired cytochrome P450 2D6 activity genotype and phenotype with therapeutic efficacy of primaquine treatment for latent Plasmodium vivax malaria. JAMA Netw Open. 2018;1:e181449.

35. Sudathip P, Kongkasuriyachai D, Stelmach R, Bisanzio D, Sine J, Sawang S, et al. The investment case for malaria elimination in Thailand: a cost-benefit analysis. Am J Trop Med Hyg. 2019;100:1445-53.

\section{Figures}

\begin{tabular}{|c|c|c|c|c|c|c|c|c|c|}
\hline \multicolumn{6}{|c|}{ Malaria Case Follow up Form (FU form) } & \multicolumn{4}{|c|}{ Patient Code: } \\
\hline \multicolumn{6}{|c|}{ Hospital/Health Service } & \multicolumn{4}{|c|}{ (Vbdu code/ Date of Davo/ Epi1 Serial no. 3diaits) } \\
\hline \multicolumn{10}{|c|}{ Date of diagnosis............................... } \\
\hline \multicolumn{10}{|c|}{ 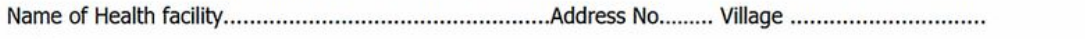 } \\
\hline \multicolumn{10}{|c|}{ 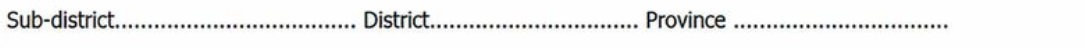 } \\
\hline \multicolumn{10}{|c|}{ Patient Information } \\
\hline \multicolumn{10}{|c|}{ Patient full Name............................................Age..........years old } \\
\hline \multicolumn{10}{|c|}{ 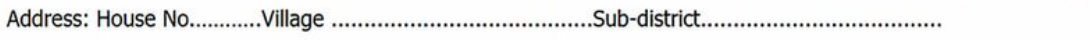 } \\
\hline \multicolumn{10}{|c|}{ 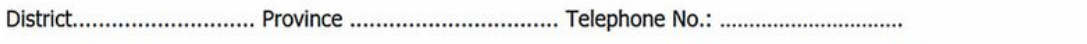 } \\
\hline \multicolumn{10}{|c|}{ National ID. : ..................................... o Epi1 serial No.: ................. ० H } \\
\hline \multicolumn{10}{|c|}{ 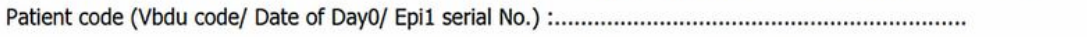 } \\
\hline \multicolumn{10}{|c|}{ Results of Diagnosis } \\
\hline \multirow{2}{*}{\multicolumn{10}{|c|}{ 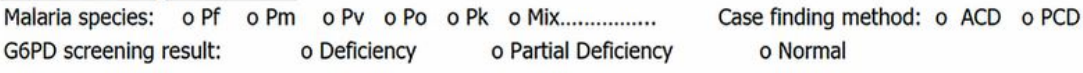 }} \\
\hline & & & & & & & & & \\
\hline \multicolumn{10}{|c|}{ Treatments } \\
\hline \multirow{2}{*}{\multicolumn{10}{|c|}{$\begin{array}{l}\text { - DHA - PIP o Children dose } 20 / 160 \mathrm{mg} \\
\text { o Chloroquine } \quad \text { Total no.of tablet.......... }\end{array}$}} \\
\hline & & & & o Pri. & ........ Mg & Total no & .of tablet... & & \\
\hline \multicolumn{10}{|c|}{ o ATS Total no.of tablet......... $\quad$ o MQ $\quad$ Total no.of tablet.......... } \\
\hline \multicolumn{10}{|c|}{ o Tetracycline Total no.of tablet.......... } \\
\hline \multirow{2}{*}{\multicolumn{10}{|c|}{ - ATS IV Total no.of vial........... }} \\
\hline \multicolumn{9}{|c|}{ ○ Atovaquone-proguanil Total no.of tablet.......... o AL Total no.of tablet.......... } & \\
\hline \multicolumn{10}{|c|}{ o Others (please specify).................................... Total no.of tablet........... } \\
\hline Monitoring date & $\begin{array}{c}\text { Do } \\
\ldots / \ldots / \ldots\end{array}$ & $\begin{array}{c}\text { D3 } \\
\ldots / \ldots / \ldots\end{array}$ & $\begin{array}{c}\text { D7 } \\
\ldots / \ldots / \ldots\end{array}$ & $\begin{array}{c}\text { D14 } \\
\ldots / \ldots / \ldots\end{array}$ & $\begin{array}{c}\text { D28 } \\
\ldots / \ldots / \ldots\end{array}$ & $\begin{array}{c}\text { D42 } \\
\ldots / \ldots / \ldots\end{array}$ & $\begin{array}{c}\text { D60 } \\
\ldots / \ldots / \ldots\end{array}$ & $\begin{array}{c}\text { D90 } \\
\ldots / \ldots / \ldots\end{array}$ & Remarks \\
\hline $\begin{array}{l}\text { Actual date patient } \\
\text { presents }\end{array}$ & $\ldots / \ldots / \ldots$ & $\ldots / \ldots / \ldots$ & $\ldots / \ldots / \ldots$ & $\ldots / \ldots / \ldots$ & $\mid \ldots / \ldots / \ldots$ & $\ldots / \ldots / \ldots$ & $\ldots / \ldots / \ldots$ & .../.../... & \\
\hline \multicolumn{10}{|l|}{ Temperature $\left({ }^{\circ} \mathrm{C}\right)$} \\
\hline \multicolumn{10}{|l|}{$\begin{array}{l}\text { No. of Parasite: No. } \\
\text { of Trophozoites/ } \\
\text { WBCs }\end{array}$} \\
\hline \multicolumn{10}{|l|}{ Side effects: } \\
\hline Date _L_ _ & O nausea 0 & vomit O heac & dache $O$ body & $y$ pains and a & aches 0 diarri & thea O skin ra & ashes 0 others & s (specify) ...... & 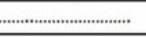 \\
\hline Date ___ _ & O nausea 0 & vomit O hea & dache $\mathrm{O}$ body & $y$ pains and a & aches 0 diarri & thea O skin ra & ashes 0 others & s (specify) ...... & 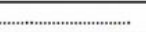 \\
\hline Date ______ & O nausea 0 & vomit O heac & dache $\mathrm{O}$ body & $y$ pains and $\mathrm{a}$ & aches O diarrt & Thea O skin ra & ashes 0 others & is (specify) ..... & 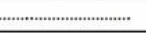 \\
\hline
\end{tabular}

\section{Managements:}

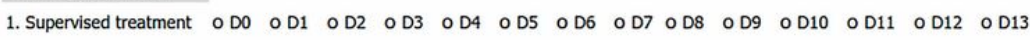

2. Does the patient vomint within 30 minutes of drug intake? o Yes o No If yes, was the second dose given? O Yes o No

3. Two slides of thick and thin films (on same

slide)

4. Filter paper with 3 dried blood spots

$\begin{array}{llllllllllllllll}\circ & D 0 & \circ & D 3 & \circ & D 7 & \circ & D 14 & \circ & D 28 & \circ & D 42 & \circ & D 60 & \circ & D 90\end{array}$

$\begin{array}{lllllllllllllllll}\circ & D 0 & \circ & D 3 & \circ & D 7 & \circ & D 14 & \circ & D 28 & \circ & D 42 & \circ & D 60 & \circ & D 90\end{array}$

Please specify reasons of incomplete or lost to follow-up

Reponsible health worker's name.

Date.

\section{Figure 1}

Malaria Case Follow-Up form 

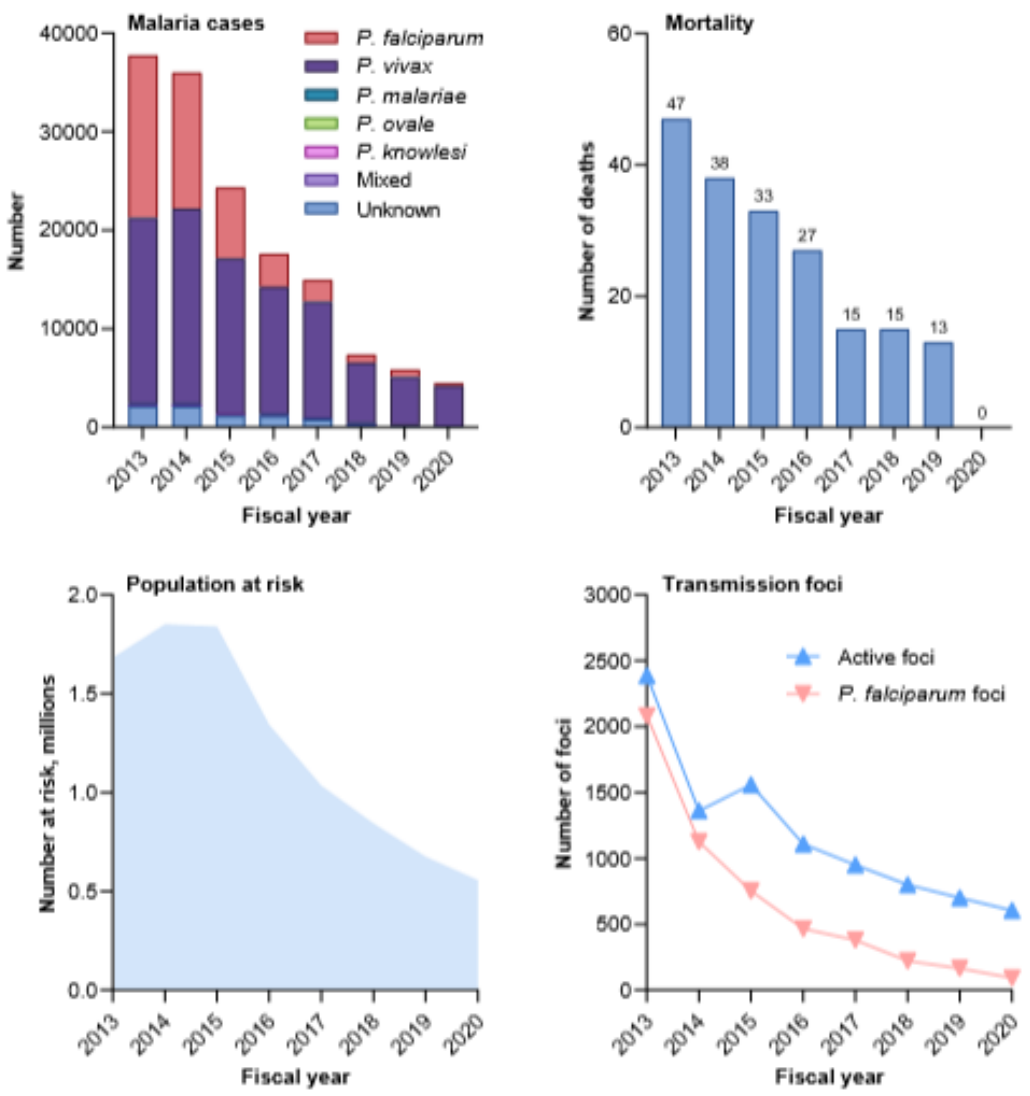

\section{Figure 2}

Malaria trends in Thailand FY2013-FY2020 Malaria mortality figures for FY2020 are provisional until officially approved by the Division of Planning and Strategy, Ministry of Public Health, Thailand. 


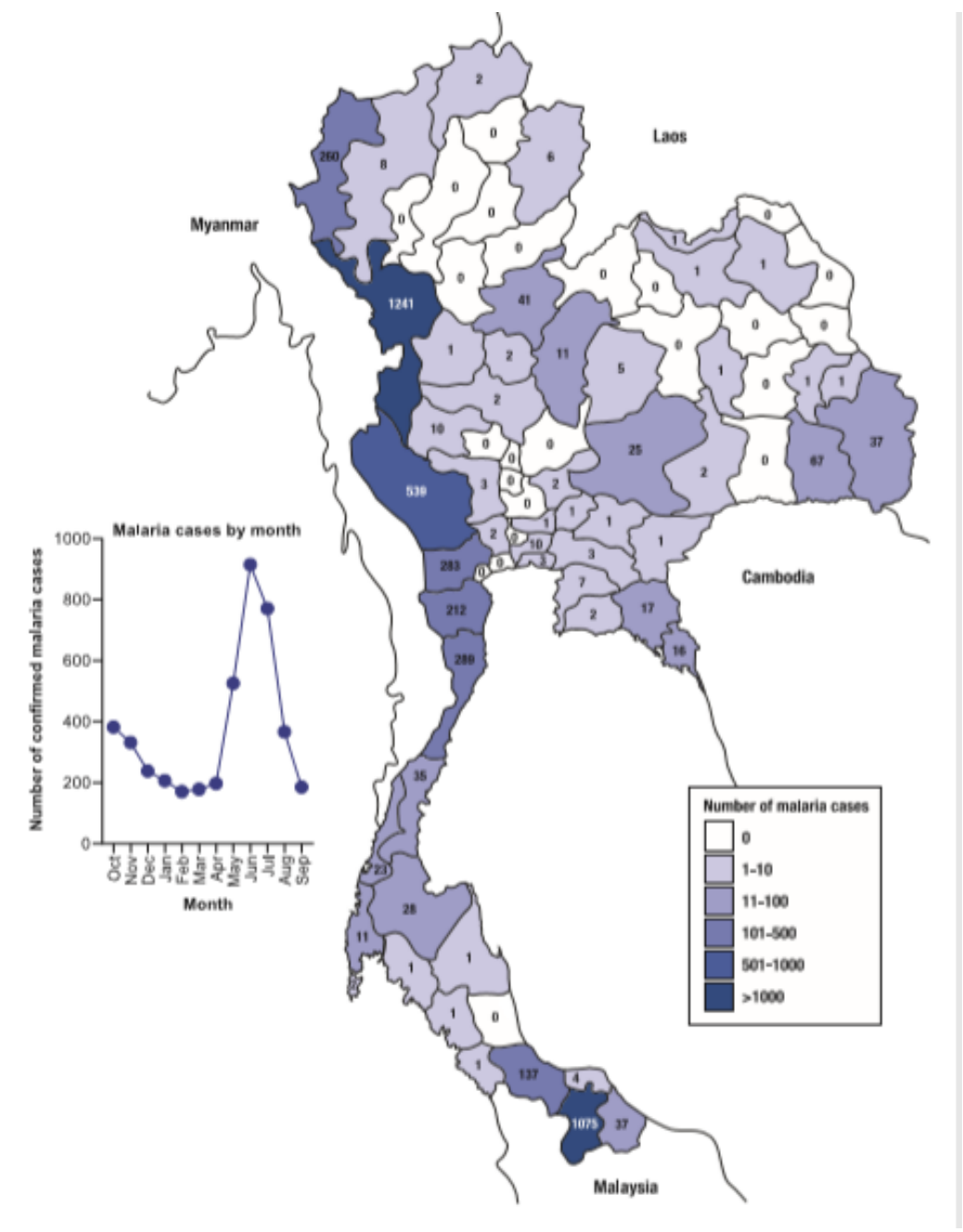

\section{Figure 3}

Temporal and geographical distribution of malaria cases in Thailand FY2020 Malaria mortality figures for FY2020 are provisional until officially approved by the Division of Planning and Strategy, Ministry of Public Health, Thailand. Note: The designations employed and the presentation of the material on this map do not imply the expression of any opinion whatsoever on the part of Research Square concerning the legal status of any country, territory, city or area or of its authorities, or concerning the delimitation of its frontiers or boundaries. This map has been provided by the authors. 
Origin of malaria infection

○०০০০০০০০০ ०००००००००० ०000000000 ०००0000000 O०OO०OO०OO ०००००००००० ०००००००००० O०OO०OO०OO

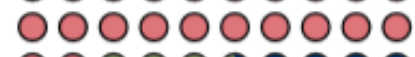
०००००००००० $\square$ Same household

$\square$ Home village

$\square$ Home district Different district Home province

$\square$ Different province

$\square$ Another country

$\square$ Other source

Unspecified

\section{Patient age}

○००००००००० ०००००००००० ०००००००००० ००००००0000 ०००००००००० O००0000000 ००00000000 OOOOOOOOOO

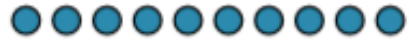
०००००००००० $\square<5$ years

$\square \geq 5$ to $<15$ years

$\square \geq 15$ to $<25$ years

$\square \geq 25$ to 45 years

$\square \geq 45$ years

\section{Patient sex}

०००००००००० ०००००००००० 0000000000 ०००००००००० ०००००००००० O००O०००००० ०००००००००० ०००००००००० ०००००००००० ०००००००००० $\square$ Male

$\square$ Female

\section{Patient residency}

०००००००००० ०००००००००० ०००००००००० ०००००००००० ०००००००००० ०००००००००० ०००००००००० ○००००००००० ०००००००००० ००००००००००

\section{Figure 4}

Characteristics of malaria cases in Thailand FY2020 Residency background was categorized as resident Thai, long-term migrant ( $\geq 6$ months residency), or short-term migrant ( $<6$ months residency). 

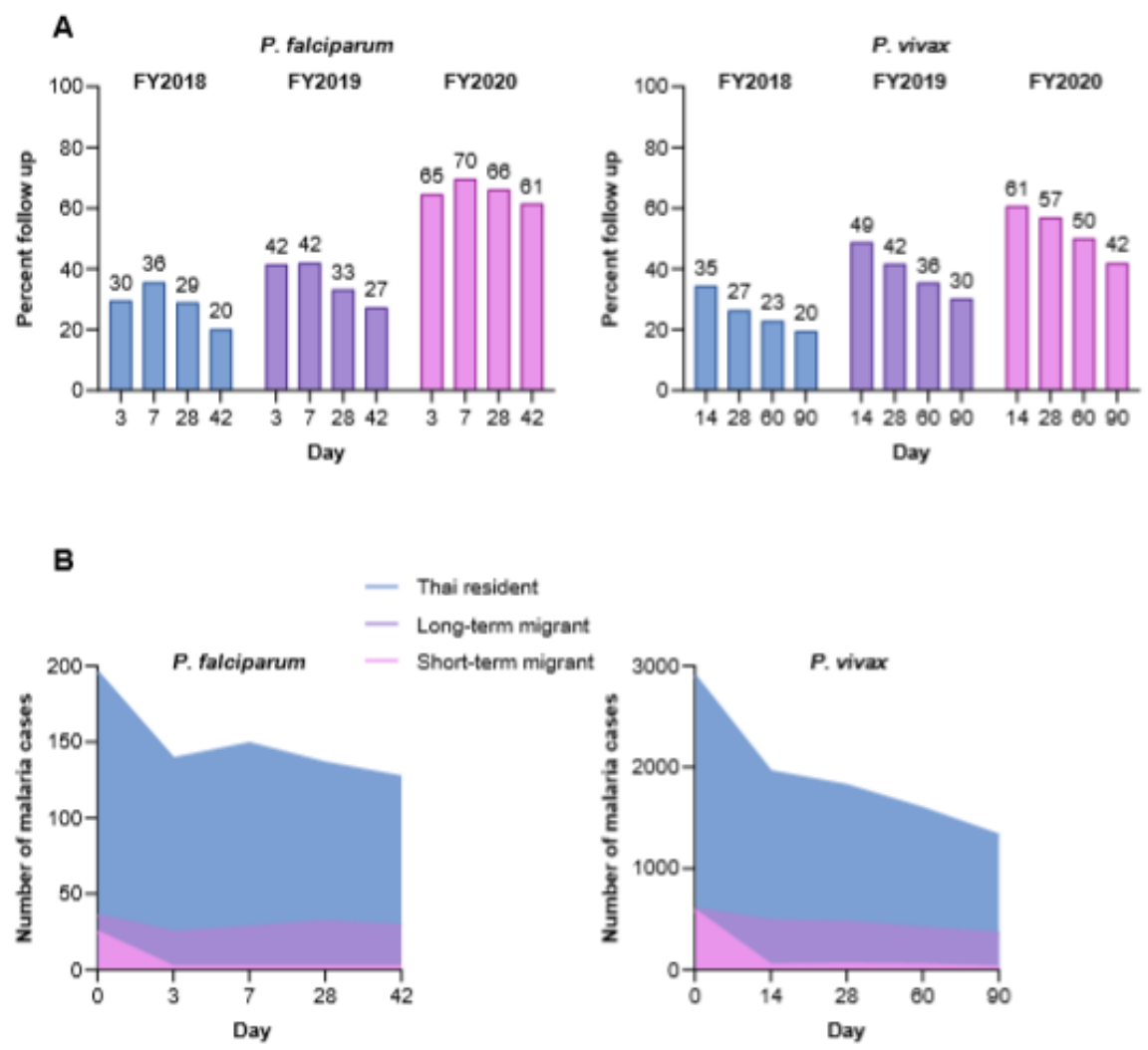

\section{Figure 5}

Malaria case follow-up rates in Thailand (A) All patients FY2018-FY2020, and (B) By patient origin in FY2020.

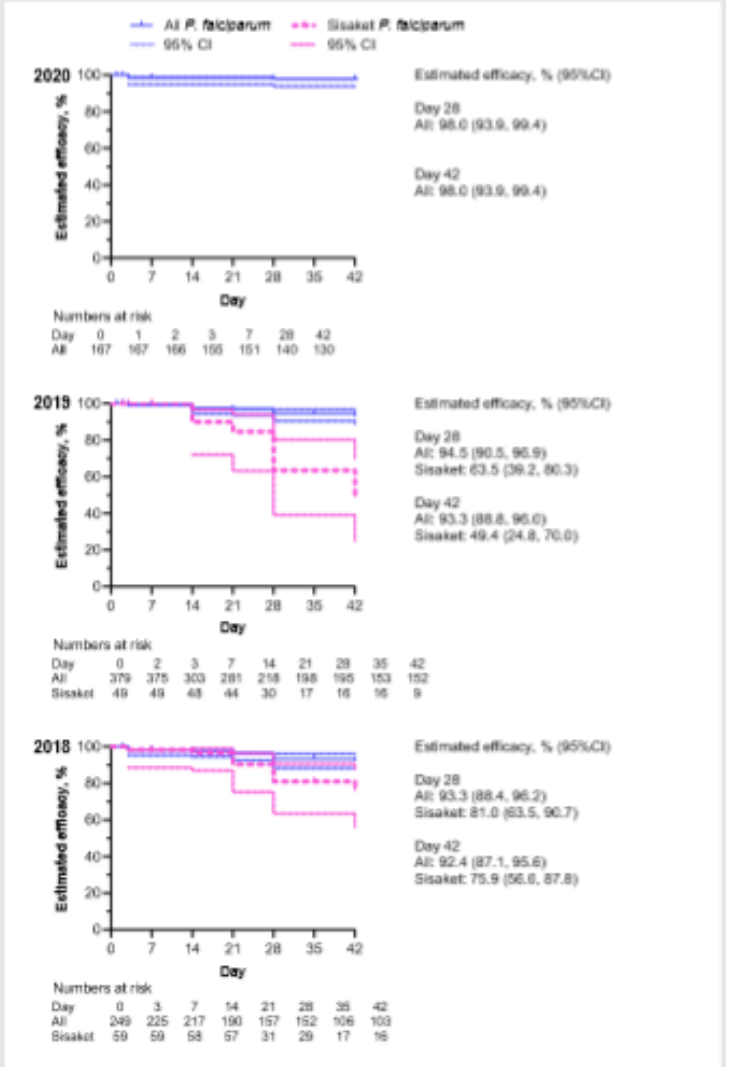

Figure 6 
Efficacy of dihydroartemisinin-piperaquine plus primaquine against P. falciparum malaria in Thailand FY2018-FY2020 Data are Kaplan-Meier estimates for patients who had P. falciparum monoinfection, received at least one dose of both dihydroartemisinin-piperaquine and primaquine, and attended at least one follow-up visit. In Sisaket Province, pyronaridineartesunate was rolled out as a new first-line treatment in FY2020, so data for this province are not shown for that year (see text).
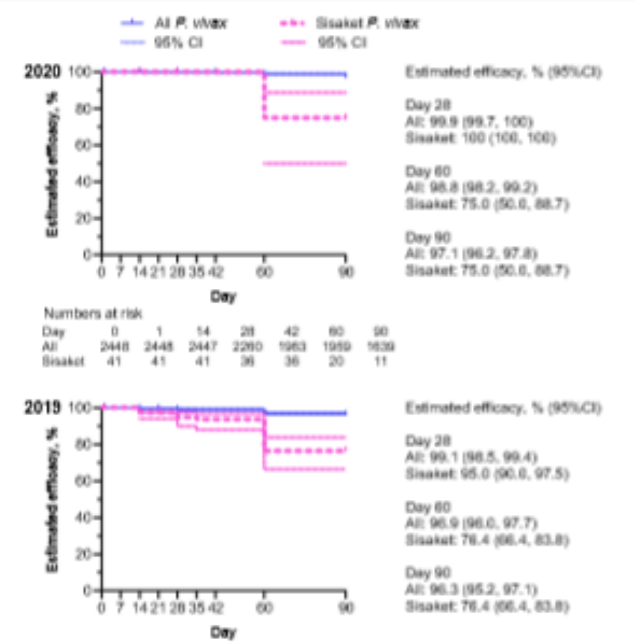

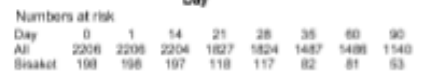

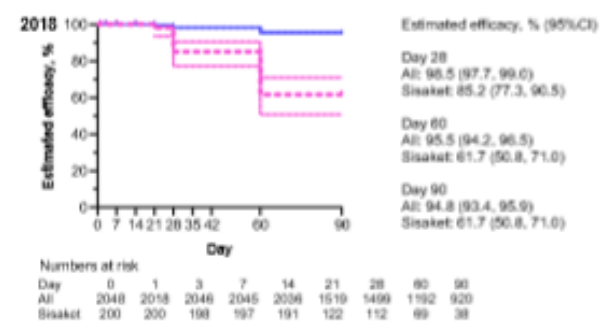

\section{Figure 7}

Efficacy of chloroquine plus primaquine for P. vivax malaria in Thailand FY2018-FY2020 Data are Kaplan-Meier estimates for patients who had P. vivax monoinfection, received at least one dose of both chloroquine and primaquine, and attended at least one follow-up visit. 